\title{
Applications of nanoparticle drug delivery systems for the reversal of multidrug resistance in cancer (Review)
}

\author{
YINGHONG HUANG ${ }^{1}$, SUSAN P.C. COLE ${ }^{2}$, TIANGE CAI ${ }^{3}$, and YU CAI $^{1,4}$ \\ ${ }^{1}$ Department of Traditional Chinese Medicine, College of Pharmacy, Jinan University, Guangzhou, \\ Guangdong 510632, P.R. China; ${ }^{2}$ Department of Pathology and Molecular Medicine, Division of Cancer Biology \\ and Genetics, Queen's University Cancer Research Institute, Kingston, ON K7L 3N6, Canada; ${ }^{3}$ Department of \\ Biological Sciences, College of Life Sciences, Liaoning University, Shenyang, Liaoning 110036; \\ ${ }^{4}$ Cancer Institute of Jinan University, Guangzhou, Guangdong 510632, P.R. China
}

Received March 28, 2015; Accepted April 29, 2016

DOI: $10.3892 / \mathrm{ol} .2016 .4596$

\begin{abstract}
Multidrug resistance (MDR) to chemotherapy presents a major obstacle in the treatment of cancer patients, which directly affects the clinical success rate of cancer therapy. Current research aims to improve the efficiency of chemotherapy, whilst reducing toxicity to prolong the lives of cancer patients. As with good biocompatibility, high stability and drug release targeting properties, nanodrug delivery systems alter the mechanism by which drugs function to reverse MDR, via passive or active targeting, increasing drug accumulation in the tumor tissue or reducing drug elimination. Given the potential role of nanodrug delivery systems used in multidrug resistance, the present study summarizes the current knowledge on the properties of liposomes, lipid nanoparticles, polymeric micelles and mesoporous silica nanoparticles, together with their underlying mechanisms. The current review aims to provide a reliable basis and useful information for the development of new treatment strategies of multidrug resistance reversal using nanodrug delivery systems.
\end{abstract}

Correspondence to: Mr. Yu Cai, Department of Traditional Chinese Medicine, College of Pharmacy, Jinan University, 601 Huangpu Avenue West, Guangzhou, Guangdong 510632, P.R. China

E-mail: caiyu8@sohu.com

Ms. Tiange Cai, Department of Biological Sciences, College of Life Sciences, Liaoning University, 66 Chongshan Mid-Road, Shenyang, Liaoning 110036, P.R. China

E-mail: caitiange@163.com

Abbreviations: MDR, multidrug resistance; SLNs, solid lipid nanoparticles; NLCs, nanostructured lipid carriers; PLN, polymer-lipid hybrid nanoparticle; MSNs, mesoporous silica nano drug delivery systems

Key words: nanoparticle drug delivery system, MDR, SLN, NLC, PLN, polymeric micelles, MSNs, liposomes, phospholipid bilayer, cancer, applications

\section{Contents}

1. Introduction

2. MDR and applications of nanodrug transmission systems

3. Liposomes

4. Lipid nanoparticles

5. Polymeric micelles

6. Combined treatment strategies based on nanodrug delivery systems

7. Mesoporous silica nanoparticles

8. Conclusion

\section{Introduction}

At present, chemotherapy remains the optimal choice for cancer therapy, and tumor multidrug resistance (MDR) is a major factor that reduces the efficacy of chemotherapy (1). MDR is a phenotype that tumor cells acquire, which confers resistance to certain chemotherapy drugs, as well as concurrent cross-resistance to additional antitumor drugs that have different structures or mechanisms of action $(2,3)$. The complexity of MDR has impeded the study of reversal agents (3-5). In recent years, the application of nanotechnology for drug carrier design has resulted in the development of novel nanoparticle drug delivery systems that aim to reverse MDR (6-8). Inorganic nanodrug delivery systems, lipid-based systems and polymer nanodrug delivery systems are the most common nanodrug delivery systems, which exhibit non-toxic, biocompatible and highly stable properties $(8,9)$. The application of nanoparticle drug delivery systems is increasing due to their advantage of controlled and targeted drug release $(9,10)$. Studies have demonstrated that entrapped small molecule drugs (10-200 $\mathrm{nm}$ in diameter) are more conducive to drug uptake and efflux; these nanodrug particles function via passive and active mechanisms, whereas in the systemic blood circulation they exhibit sustained release that subsequently enhances intracellular drug accumulation in tumor cells, yielding an improved effect (1,11-14). In the present review, the application of nanoparticle drug delivery systems in reversing the MDR of tumors is reviewed, which may provide an improved understanding of novel strategies for cancer therapy. 


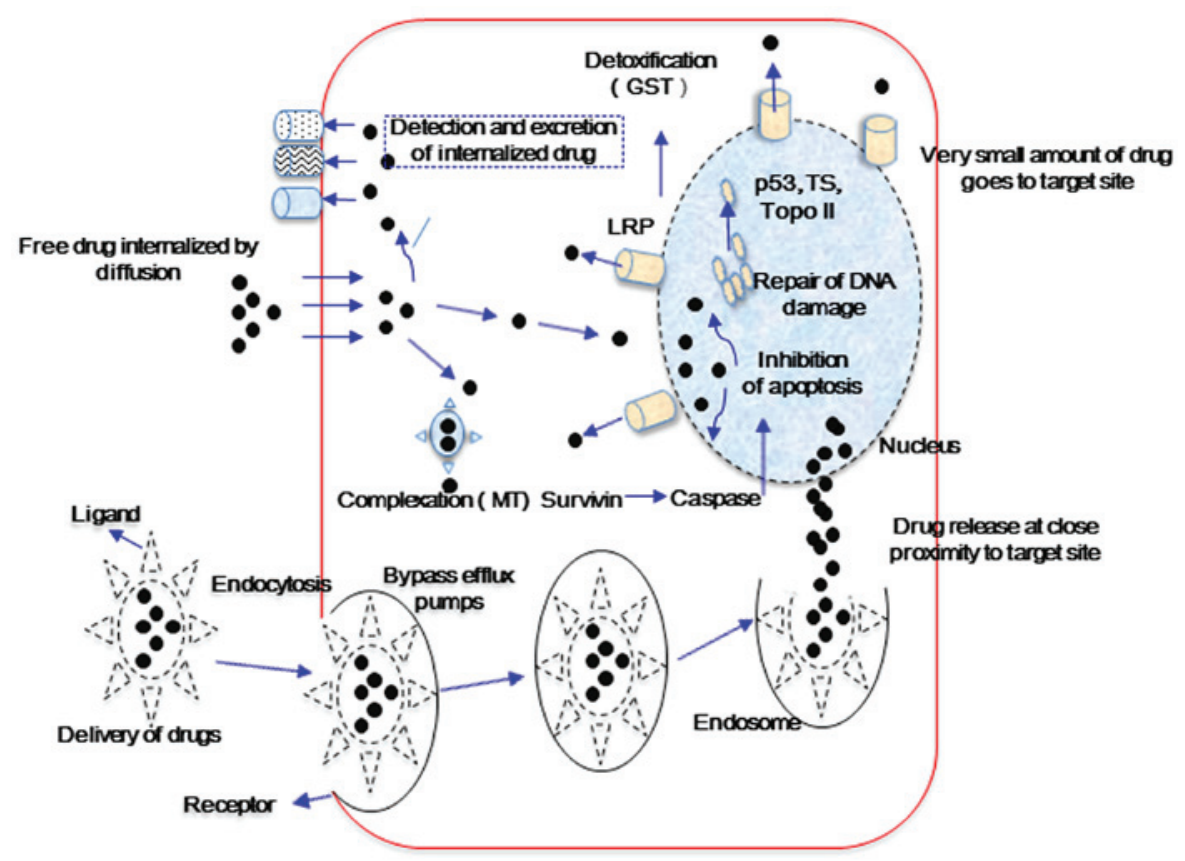

Figure 1. Main factors responsible for drug excretion and the delivery of encapsulated and free drugs. GST, glutathione transferase; Topo II, topoisomerase II; TS, thymidylate synthase; MT, metallothionein; LRP, lung resistance-related protein.

\section{MDR and applications of nanodrug transmission sys- tems}

Cancer MDR may be caused by a number of complex factors, including ATP-binding cassette (ABC) transporters, multi-drug resistance protein [P-glycoprotein 1 (P-gp)], MDR-associated proteins (MRPs), breast cancer resistance protein $(\mathrm{ABCG})$, glutathione transferase, metallothionein, DNA topoisomerase II and catalytic enzymes $(2,3,5)$. These substances exhibit various functions in the induction of tumor cell MDR (Fig. 1). Cellular MDR is divided into the ATP-dependent efflux pumps and non-ATP-dependent efflux pumps types. The 'pumps' type use the energy obtained from ATP hydrolysis to efflux drugs via ATP-dependent transport proteins, such as P-gp, MRPs and BCRPs, which decreases the intracellular drug concentration and subsequently results in drug resistance. The 'non pumps' type do not depend on the energy from ATP hydrolysis while activating the anti-apoptotic proteins, such as Bcl-2, and efflux drugs directly $(2,7,9)$. Nanoparticle drug transmission systems may alter drug uptake in tumor cells (12). The system delivers the chemotherapy drug directly to tumor cells, which functions to reverse MDR (10). Entrapment affects the pharmacokinetic properties of drugs; free drugs cross the cell membrane passively and are easily identified by the efflux pumps located on the cell membrane or captured by $\mathrm{ABC}$ transporter proteins, whereas drug-loading nanoparticles may avoid recognition by the $\mathrm{ABC}$ efflux pumps and endocytosis by ABC transporters, leading to increased intracellular accumulation of chemotherapeutic drugs (Fig. 1) (10,12,14).

\section{Liposomes}

Liposomes are preparations that utilize a phospholipid bilayer membrane vesicle to encapsulate drug molecules. The hydrophilic and hydrophobic bilayer cores entrap hydrophilic and lipophilic drugs, respectively. Liposomes have been demonstrated to prolong the blood-circulation time of drugs, to alter the pharmacokinetics and distribution of P-gp inhibitors in vivo, and to increase the drug concentration in the tumor cells, while reducing the impact on normal tissues, thus exerting toxicity to enhance the effects of chemotherapy (15-21). A study by Zhou et al (22), which investigated MDR reversal using doxorubicin (DOX) liposomes in vitro, demonstrated that DOX liposomes were mainly detected in the nucleus of human breast cancer P-gp overexpression cells (MCF-7/Adr) with an increased toxicity, and exhibited a stronger cellular retention capacity in human carcinoma KBv200 cells. Kang et al (23) used rhodamine (a P-gp substrate) to penetrate liposomes, and this combination resulted in increased liposome retention in the MCF-7/Adr cell line, which exhibits P-gp overexpression in vitro. Further assessment demonstrated that the incorporation of cholesterol and polyethyleneglycol-attached lipids was effective in further increasing the rhodamine retention in MCF-7/Pgp cells. More rigid liposomes are able to sequester rhodamine more efficiently, thereby inhibiting direct interactions of rhodamine with P-gp proteins. These studies indicated that by optimizing the composition of liposomes, reduction of P-gp-mediated MDR may be achieved.

\section{Lipid nanoparticles}

Lipid nanoparticles are a type of nanodrug delivery system that are assembled using natural or synthetic lipids, such as stearic acid, lecithin and triglycerides, as the matrix, to entrap anticancer drugs (particle size, 50-1,000 nm) in a lipid core $(23,24)$. Lipid nanoparticles have various routes of administration, exhibit good biological compatibility and stability, and effectively control drug release to avoid degradation and leakage. Solid lipid nanoparticles (SLNs) and nanostructured 
lipid carriers (NLCs) are the most common types, which have gained increasing attention as they represent promising drug carriers $(25,26)$. One study showed that NLCs exhibit increased stability when compared with SLNs (24). Wong et al (27) showed that, compared with free doxorubicin, DOX-loaded SLNs exhibit a high encapsulation efficiency, a faster release rate in vitro, and an enhanced uptake and retention capacity in human breast cancer cells (MDA435/LCC6/MDR1), resulting in significantly enhanced cytotoxicity. A novel polymer-lipid hybrid nanoparticle (PLN) system has also been developed (28). Prepared DOX-GG918-PLN and doxorubicin and mitomycin C co-loaded PLN carriers exhibited a good antitumor effect in vitro and in vivo, with significant reversal effects, which indicates that the carriers present a novel technique for improving the efficacy of local solid tumor chemotherapy (29-32). Liposomes alone exhibit no specific targeting, however, via the modification of nanodrug delivery systems using various ligands, including folic acid or anti-transferrin monoclonal antibodies, specific targeting may be obtained (33-35). Ligands with selective and specific affinities may be incorporated into the lipid bilayer of liposomes, which may then be identified by specific cells, yielding targeted nanoparticles $(36,37)$.

\section{Polymeric micelles}

Polymeric micelles, which are a type of drug carrier with a hydrophobic core and hydrophilic polyethylene glycol (PEG) shell, present an ideal choice for the effective delivery of anticancer drugs (particle size, 10-100 nm) with poor solubility $(38,39)$. Compared with the free drug, drug-loaded polymeric micelles, which demonstrate a higher stability and biocompatibility in vitro and vivo, and a significantly increased blood circulation time, are transferred to the tumor site via active and passive targeting mechanisms. Passive targeting occurs via the enhanced permeability and retention effect, which causes drugs to be selectively accumulated and released at the tumor site. By modifying the surface of polymer micelles, drug accumulation by the tumor may be increased via the active targeting pathway. Therefore, polymer micelles present an ideal carrier molecule for active or passive targeted drug delivery as they improve the therapeutic index while reducing toxic side effects (40-44). The use of drug-conjugated polymer micelles that exhibit simultaneous entrapment of two drugs and maintain antitumor activity has been reported (38). Currently, several types of polymer micelle transmission systems are being used to overcome MDR, and certain studies have demonstrated that a number of block copolymers may decrease the production of ATP in resistant cells, thus reducing the activity of P-gp (45-47). Saiyin et al (48) implemented the simultaneous entrapment of a chemotherapeutic drug and autophagy inhibitors, and engineered the sequential release of these drugs. The prioritized release of the autophagy inhibitors suppressed the phagocytosis of tumor cells, subsequently increasing sensitivity to the chemotherapeutic drug, which resulted in a synergistic effect. This combination provides a novel platform for chemotherapy. Yu et al (49) prepared docetaxel-loaded pH-responsive PEG-hyperbranched polyacylhydrazone micelles, which exhibited increasing tumor toxicity in vitro; however, when combined with glucose, enhanced antitumor activity and lower systemic toxicity were observed. Therefore, these results indicate that polymeric micelles may be used for selective targeted drug delivery.

\section{Combined treatment strategies based on nanodrug de- livery systems}

Enhancing the efficiency of drug delivery to tumor cells and preventing apoptosis by modulating intracellular signal transduction mechanisms presents an effective method to overcome MDR (50-52). The co-delivery of reversal agents and chemotherapy drugs by nanodrug delivery systems may regulate the expression of intracellular ABC transporter proteins and silence MDR genes (52-54), thereby affecting the efflux or apoptosis of tumor cells and reducing the toxicity of chemotherapy (55-57). In a study by Abouzeid et al (58), co-coated curcumin (a reversal agent) and paclitaxel-loaded PEG-phosphatidylethanolamine/vitamin E micelles were demonstrated to exhibit significantly increased toxicity in human ovarian carcinoma resistant cells (SK-OV-3/TR) in vitro and in vivo, with the synergistic antitumor effects observed in vivo being superior to those observed in vitro. Furthermore, Tang et al (59) reported that the co-delivery of DOX and a P-gp inhibitor (verapamil) using a reduction-sensitive liposome resulted in increased apoptosis induction and necrosis of MCF-7/Adr tumor cells.

\section{Mesoporous silica nanoparticles}

Recently, gene technology and nanobiomaterial vector delivery systems, such as silicon nanogene vectors as non-viral gene vectors, have gained increasing attention as a promising approach for tumor MDR reversal (60-63). Silicon nanodrug systems exhibit good biocompatibility and a non-cytotoxic surface that is easily modified. The main aims of the formed mesoporous silica nanodrug delivery systems (MSNs) are to control targeted-drug release and overcome MDR (64-66). With regard to MDR reversal, MSNs are considered to alter the original route of drug uptake, while avoiding identification, binding and efflux by $\mathrm{ABC}$ proteins. In addition, the different MSN pore sizes allow the effectively controlled release of intratumor drugs and accumulation (67-70). MSNs present an alternative method for the more efficient delivery of chemotherapy drugs and targeted gene therapy. Meng et al (70) revealed that coating $\mathrm{P}$-gp-targeted siRNA with DOX using MSNs significantly increased the chemotherapeutic drug concentration within the cell nucleus of the squamous carcinoma resistant cell line, KB-V1, and promoted cell apoptosis and death. Notably, Zhang et al (71) also developed a polymer-lipid supported-MSN. This system exhibited targeted and controlled drug release in vitro and exhibited a significant effect in the treatment of breast cancer, with a low toxicity in vivo.

\section{Conclusion}

Cancer is a common disease that seriously affects human health and reversal of MDR in cancer has long been a topic of research. However, due to the complex and diverse resistance mechanisms of cancer, the limitation of biological activity and 
toxicity of MDR reversal agents, the existing reversal agents cannot meet the requirements of treatment. Compared with traditional anticancer drugs, nanodrug delivery systems show a clear advantage in terms of reversing MDR during the process of tumor therapy. Novel drug-loaded nanoparticle systems, such as the co-entrapped reversal agents and chemotherapy drugs and the co-loaded P-gp inhibitors and chemotherapy drugs, and the specific modification of nanoparticles may alter the transmission method and the targeting of drugs and antagonize the drug efflux by tumor cells. Thus, the accumulation of drug within the tumor cells may be improved. The good biocompatibility, high stability, drug release and targeting of nanodrug delivery systems overcome the shortcomings of the traditional drug delivery system, and show great promise in cancer MDR reversal. However, with the enhanced targeting, the tissue distribution of drugs has also changed and led to new adverse reactions; therefore, additional in vivo evaluation of safety and efficacy data are urgently required.

\section{Acknowledgements}

This study was supported by the National Natural Science Foundation of China (grant nos. 81273707 and 81173215), the Ministry of Education in the New Century Excellent Talents (grant no. NECT-12-0677), the Natural Science Foundation of Guangdong (grant nos.S2013010012880 and 2016A030311037), the Science and Technology Program of Guangzhou (grant no. 2014J4500005), the Science Program of the Department of Education of Guangdong (grant nos. 2013KJCX0021 and 2015KGJHZ012) and the Science and Technology Program of Guangdong (grant no. 2015A050502027).

\section{References}

1. Gottesman MM, Fojo T and Bates SE: Multidrug resistance in cancer: Role of ATP-dependent transporters. Nat Rev Cancer 2: 48-58, 2002.

2. Gottesman MM and Ling V: The molecular basis of multidrug resistance in cancer: The early years of P-glycoprotein research. FEBS Lett 580: 998-1009, 2006.

3. Choi CH: ABC transporters as multidrug resistance mechanisms and the development of chemosensitizers for their reversal. Cancer Cell Int 5: 30, 2005.

4. Noguchi K, Katayama K, Mitsuhashi J and Sugimoto Y: Functions of the breast cancer resistance protein (BCRP/ABCG2) in chemotherapy. Adv Drug Deliv Rev 61: 26-33, 2009

5. Sugimoto Y, Tsukahara S, Ishikawa E and Mitsuhashi J: Breast cancer resistance protein: Molecular target for anticancer drug resistance and pharmacokinetics/pharmacodynamics. Cancer Sci 96: 457-465, 2005.

6. Wang F, Wang YC, Dou S, Xiong MH, Sun TM and Wang J: Doxorubicin-tethered responsive gold nanoparticles facilitate intracellular drug delivery for overcoming multidrug resistance in cancer cells. ACS Nano 5: 3679-3692, 2011.

7. Diao YY, Han M, Chen DW and Gao JQ: Progress in the study of micelle delivery system reversing multidrug resistance. Yao Xue Xue Bao 44: 710-715, 2009 (In Chinese).

8. Kapse-Mistry S, Govender T, Srivastava R and Yergeri M: Nanodrug delivery in reversing multidrug resistance in cancer cells. Front Pharmacol 5: 159, 2014.

9. Hu CM and Zhang L: Therapeutic nanoparticles to combat cancer drug resistance. Curr Drug Metab 10: 836-841, 2009.

10. Chidambaram M, Manavalan R and Kathiresan K: Nanotherapeutics to overcome conventional cancer chemotherapy limitations. J Pharm Pharm Sci 14: 67-77, 2011.

11. Cho K, Wang X, Nie S, Chen ZG and Shin DM: Therapeutic nanoparticles for drug delivery in cancer. Clin Cancer Res 14: 1310-1316, 2008
12. Wang J, Sui M and Fan W: Nanoparticles for tumor targeted therapies and their pharmacokinetics. Curr Drug Metab 11: 129-141, 2010.

13. Markman JL, Rekechenetskiy A, Holler E and Ljubimova JY: Nanomedicine therapeutic approaches to overcome cancer drug resistance. Adv Drug Deliv Rev 65: 1866-1879, 2013.

14. Kirtane AR, Kalscheuer SM and Panyam J: Exploiting nanotechnology to overcome tumor drug resistance: Challenges and opportunities. Adv Drug Deliv Rev 65: 1731-1747, 2013.

15. Lao J, Madani J, Puértolas T, Alvarez M, Hernández A, Pazo-Cid R, Artal A and Antón Torres A: Liposomal doxorubicin in the treatment of breast cancer patients: A review. J Drug Deliv 2013: 456409, 2013.

16. Rivera E: Liposomal anthracyclines in metastatic breast cancer: Clinical update. Oncologist 8 (Suppl 2): 3-9, 2003.

17. Lorusso V, Manzione L and Silvestris N: Role of liposomal anthracyclines in breast cancer. Ann Oncol 18 (Suppl 6): vi70-vi73, 2007.

18. Arias JL, Clares B, Morales ME, Gallardo V and Ruiz MA: Lipid-based drug delivery systems for cancer treatment. Curr Drug Targets 12: 1151-1165, 2011.

19. Samad A, Sultana Y and Aqil M: Liposomal drug delivery systems: An update review. Curr Drug Deliv 4: 297-305, 2007.

20. Lian T and Ho RJ: Trends and developments in liposome drug delivery systems. J Pharm Sci 90: 667-680, 2001.

21. Goyal P, Goyal K, Vijaya Kumar SG, Singh A, Katare OP and Mishra DN: Liposomal drug delivery systems-clinical applications. Acta Pharm 55: 1-25, 2005.

22. Zhou WJ, Zhou W, Jia SW, et al: The cytology evaluation of doxorubicin liposomes to overcome multidrug resistance in vitro. Zhong Guo Yi Yuan Yao Xue Za Zhi 32: 1349-1352, 2012 (In Chinese).

23. Kang DI, Kang HK, Gwak HS, Han HK and Lim SJ: Liposome composition is important for retention of liposomal rhodamine in p-glycoprotein-overexpressing cancer cells. Drug Deliv 16: 261-267 2009.

24. Shidhaye SS, Vaidya R, Sutar S, Patwardhan A and Kadam VJ: Solid lipid nanoparticles and nanostructured lipid carriers-innovative generations of solid lipid carriers. Curr Drug Deliv 5: 324-331, 2008.

25. Li XW, Sun LX, Lin XH and Zeng LQ: Solid lipid nanoparticles as drug delivery system. Prog Chem 19: 87-92, 2007.

26. Xu DH, Gao JQ, Liang WQ, Yang JM and Yao Q: Progress of solid lipid nanoparticles in overcoming multidrug resistance. Zhong Guo Yao Xue Za Zhi 45: 401-403, 2010.

27. Wong HL, Bendayan R, Rauth AM and Wu XY: Development of solid lipid nanoparticles containing ionically complexed chemotherapeutic drugs and chemosensitizers. J Pharm Sci 93: 1993-2008, 2004.

28. Wong HL, Rauth AM, Bendayan R, Manias JL, Ramaswamy M, Liu Z, Erhan SZ and Wu XY: A new polymer-lipid hybrid nanoparticle system increases cytotoxicity of doxorubicin against multidrug-resistant human breast cancer cells. Pharm Res 23: 1574-1585, 2006.

29. Wong HL, Rauth AM, Bendayan $\mathrm{R}$ and $\mathrm{Wu} X Y$ : In vivo evaluation of a new polymer-lipid hybrid nanoparticle (PLN) formulation of doxorubicin in a murine solid tumor model. Eur $\mathrm{J}$ Pharm Biopharm 65: 300-308, 2007.

30. Wong HL, Bendayan R, Rauth AM and Wu XY: Simultaneous delivery of doxorubicin and GG918 (Elacridar) by new polymer-lipid hybrid nanoparticles (PLN) for enhanced treatment of multidrug-resistant breast cancer. J Control Release 116: 275-284, 2006

31. Shuhendler AJ, Prasad P, Zhang RX, Amini MA, Sun M, Liu PP, Bristow RG, Rauth AM and Wu XY: Synergistic nanoparticulate drug combination overcomes multidrug resistance, increases efficacy, and reduces cardiotoxicity in a nonimmunocompromised breast tumor model. Mol Pharm 11: 2659-2674, 2014.

32. Prasad P, Cheng J, Shuhendler A, Rauth AM and Wu XY: A novel nanoparticle formulation overcomes multiple types of membrane efflux pumps in human breast cancer cells. Drug Deliv Transl Res 2: 95-105, 2012.

33. Miao J, Du YZ, Yuan H, Zhang XG and Hu FQ: Drug resistance reversal activity of anticancer drug loaded solid lipid nanoparticles in multi-drug resistant cancer cells. Colloids Surf B Biointerfaces 110: 74-80, 2013.

34. Bourseau-Guilmain E, Béjaud J, Griveau A, Lautram N, Hindré F, Weyland M, Benoit JP and Garcion E: Development and characterization of immuno-nanocarriers targeting the cancer stem cell marker ac133. Int J Pharm 423: 93-101, 2012. 
35. Sahoo SK and Labhasetwar V: Enhanced antiproliferative activity of transferrin-conjugated paclitaxel-loaded nanoparticles is mediated via sustained intracellular drug retention. Mol Pharm 2: 373-383, 2005.

36. Zhang MZH, Chen HG and Han J: Advances in drug carrier to overcome mdr. Jie Fang Jun Yao Xue Xue Bao 30: 256-259, 2014

37. Ye G, Ke AW and Li X: The mrk-16 modified immune doxorubicin liposome in reversing multidrug resistance of cancer cells. Journal of Modern Oncology 15: 754-757, 2007.

38. Zhang Y, Huang Y and Li S: Polymeric micelles: Nanocarriers for cancer-targeted drug delivery. AAPS PharmSciTech 15 862-871, 2014.

39. Tian Y and Mao S: Amphiphilic polymeric micelles as the nanocarrier for peroral delivery of poorly soluble anticancer drugs Expert Opin Drug Deliv 9: 687-700, 2012.

40. Croy SR and Kwon GS: Polymeric micelles for drug delivery. Curr Pharm Des 12: 4669-4684, 2006.

41. Adams ML, Lavasanifar A and Kwon GS: Amphiphilic block copolymers for drug delivery. J Pharm Sci 92: 1343-1355, 2003.

42. Han SS, Li ZY, Zhu JY, Han K, Zeng ZY, Hong W, Li WX, Jia HZ Liu Y, Zhuo RX and Zhang XZ: Dual-pH sensitive charge-reversal polypeptide micelles for tumor-triggered targeting uptake and nuclear drug delivery. Small 11: 2543-2554, 2015.

43. Torchilin VP: Passive and active drug targeting: Drug delivery to tumors as an example. Handb Exp Pharmacol 197: 3-53, 2010

44. Kedar U, Phutane P, Shidhaye S and Kadam V: Advances in polymeric micelles for drug delivery and tumor targeting. Nanomedicine 6: 714-729, 2010.

45. Prabhu RH, Patravale VB and Joshi MD: Polymeric nanoparticles for targeted treatment in oncology: Current insights. Int J Nanomedicine 10: 1001-1018, 2015.

46. Mao SR, Tian Y and Wang LL: Advances in drug nanocarriers: Polymer micelles. Shenyang Yao Ke Da Xue Xue Bao 27: 979-986, 2010.

47. Zhang HJ, Zhang C and Ping QN: Research and application of polymeric micelles as a pharmaceutically acceptable carrier. Yao Xue Jin Zhan 26: 326-329, 2002.

48. Saiyin W, Wang D, Li L, Zhu L, Liu B, Sheng L, Li Y, Zhu B, Mao L, Li G and Zhu X: Sequential release of autophagy inhibitor and chemotherapeutic drug with polymeric delivery system for oral squamous cell carcinoma therapy. Mol Pharm 11: 1662-1675, 2014.

49. Yu J, Deng H, Xie F, Chen W, Zhu B and Xu Q: The potential of pH-responsive PEG-hyperbranched polyacylhydrazone micelles for cancer therapy. Biomaterials 35: 3132-3144, 2014.

50. Ganta S and Amiji M: Coadministration of paclitaxel and curcumin in nanoemulsion formulations to overcome multidrug resistance in tumor cells. Mol Pharm 6: 928-939, 2009.

51. Katsman A, Umezawa $K$ and Bonavida B: Chemosensitization and immunosensitization of resistant cancer cells to apoptosis and inhibition of metastasis by the specific NF-kappaB inhibitor DHMEQ. Curr Pharm Des 15: 792-808, 2009.

52. Yadav S, Van Vlerken LE, Little SR and Amiji MM: Evaluations of combination MDR-1 gene silencing and paclitaxel administration in biodegradable polymeric nanoparticle formulations to overcome multidrug resistance in cancer cells. Cancer Chemothe Pharmacol 63: 711-722, 2009.

53. Iyer AK, Singh A, Ganta S and Amiji MM: Role of integrated cancer nanomedicine in overcoming drug resistance. Adv Drug Deliv Rev 65: 1784-1802, 2013

54. Li J, Wang Y, Zhu Y and Oupický D: Recent advances in delivery of drug-nucleic acid combinations for cancer treatment. J Control Release 172: 589-600, 2013.
55. $\mathrm{Hu} \mathrm{ZX}$ and $\mathrm{Fu} \mathrm{XD}$ : The progress of nanoparticles drug delivery system in mdr of tumor and its reversal. Zhong Guo Yi Yuan Yao Xue Za Zhi 31: 1724-1727, 2011.

56. Chen JN, Shen Q and Li SS: Progress in the study of drug delivery system based on nanoparticles to overcome multi-drug resistance. Yao Xue Xue Bao 44: 333-337, 2009 (In Chinese).

57. Qin Y, Li M, Qiu Q, Pi JT and Chen HY: The application of nanoparticles drug delivery system in multi-drug resistance of tumor. Xian Dai Sheng Wu Yi Xue Jin Zhan 14: 5564, 5599-5600, 2014.

58. Abouzeid AH, Patel NR and Torchilin VP: Polyethylene glycol-phosphatidylethanolamine (PEG-PE)/vitamin e micelles for co-delivery of paclitaxel and curcumin to overcome multi-drug resistance in ovarian cancer. Int J Pharm 464: 178-184, 2014.

59. Tang J, Zhang L, Gao H, Liu Y, Zhang Q, Ran R, Zhang Z and $\mathrm{He} \mathrm{Q}$ : Co-delivery of doxorubicin and P-gp inhibitor by a reduction-sensitive liposome to overcome multidrug resistance, enhance anti-tumor efficiency and reduce toxicity. Drug Deliv: $1-14,2015$.

60. Wang X, Teng ZG, Huang XY and Lu GM: Mesoporous silica nanoparticles for cancer theranostic drug delivery. Yao Xue Xue Bao 48: 8-13, 2013 (In Chinese).

61. Jiang XX, Yang H and Yang Y: Silicon nanometer carrier with rna interference plasmid in reversing mdr in human colon cancer. Practical Preventive Medicine 20: 1385-1389, 2013.

62. Hom C, Lu J, Liong M, Luo H, Li Z, Zink JI and Tamanoi F: Mesoporous silica nanoparticles facilitate delivery of siRNA to shutdown signaling pathways in mammalian cells. Small 6: $1185-1190,2010$

63. Slowing II, Vivero-Escoto JL, Wu CW and Lin VS: Mesoporous silica nanoparticles as controlled release drug delivery and gene transfection carriers. Adv Drug Deliv Rev 60: 1278-1288, 2008.

64. Zhao Y, Vivero-Escoto JL, Slowing II, Trewyn BG and Lin VS: Capped mesoporous silica nanoparticles as stimuli-responsive controlled release systems for intracellular drug/gene delivery. Expert Opin Drug Deliv 7: 1013-1029, 2010.

65. Pan L, Liu J, He Q, Wang L and Shi J: Overcoming multidrug resistance of cancer cells by direct intranuclear drug delivery using TAT-conjugated mesoporous silica nanoparticles. Biomaterials 34: 2719-2730, 2013

66. He Q and Shi J: MSN anti-cancer nanomedicines: Chemotherapy enhancement, overcoming of drug resistance and metastasis inhibition. Adv Mater 26: 391-411, 2014.

67. Vivero-Escoto JL, Slowing II, Trewyn BG and Lin VS: Mesoporous silica nanoparticles for intracellular controlled drug delivery. Small 6: 1952-1967, 2010.

68. Tang F, Li L and Chen D: Mesoporous silica nanoparticles: Synthesis, biocompatibility and drug delivery. Adv Mater 24: 1504-1534, 2012.

69. Gao Y, Chen Y, Ji X, He X, Yin Q, Zhang Z, Shi J and Li Y: Controlled intracellular release of doxorubicin in multidrug-resistant cancer cells by tuning the shell-pore sizes of mesoporous silica nanoparticles. ACS Nano 5: 9788-9798, 2011.

70. Meng $\mathrm{H}$, Liong M, Xia T, Li Z, Ji Z, Zink JI and Nel AE: Engineered design of mesoporous silica nanoparticles to deliver doxorubicin and P-glycoprotein siRNA to overcome drug resistance in a cancer cell line. ACS Nano 4: 4539-4550, 2010.

71. Zhang X, Li F, Guo S, Chen X, Wang X, Li J and Gan Y: Biofunctionalized polymer-lipid supported mesoporous silica nanoparticles for release of chemotherapeutics in multidrug resistant cancer cells. Biomaterials 35: 3650-3665, 2014. 